\title{
Median arcuate ligament syndrome: A clinical dilemma
}

$\mathrm{C}$ OMPRESSION of the celiac artery was first described in 1917 by Lipshutz, ${ }^{1}$ but it took almost 50 years to understand any clinical implication.

The triad of epigastric abdominal pain exacerbated by eating, weight loss, and celiac artery compression was initially described in 1963 by Harjola, ${ }^{2}$ leading to the first description of median arcuate ligament syndrome (MALS).

To this day, controversy persists around the pathology of this syndrome, with some groups labeling it a vascular disease, while others consider it a neurogenic disease.

See related article, page 140

Initial treatment was through an open surgical approach, in which the main objective was celiac artery revascularization. The inevitable consequence of this operation was a complete neurolysis performed during exposure of the vessels. This neurolysis is now believed to be the main technical aspect that results in symptom improvement, rather than revascularization. Over the last decade, experience with minimally invasive approaches has grown, and our understanding of the disease has highlighted the role of the celiac plexus nerve fibers as the most relevant anatomic structure related to this syndrome.

\section{A CHALLENGING DIAGNOSIS}

Diagnosis remains the most challenging step in treating patients with MALS. Despite growing clinical experience, most published data are limited to case reports and small caseseries with limited follow-up. There is no consensus on diagnostic criteria, and surgery does

doi:10.3949/ccjm.88a.21001 not always result in symptom resolution, with most series reporting between $75 \%$ and $85 \%$ clinical success. ${ }^{3}$

\section{Recognizing clues}

The first suspicion of MALS usually arises when imaging studies reveal stenosis of the celiac artery. Typically, most patients had computed tomography as part of their evaluation for chronic abdominal pain. Cross-sectional imaging can show narrowing of the celiac artery and exclude atherosclerotic disease as a possible etiology.

But a static image is usually not entirely diagnostic. Variation of flow during inspiration and expiration has proven to be one of the most reliable modalities to document true compression with hemodynamic consequences. Peak systolic velocities of more than $249 \mathrm{~cm} / \mathrm{sec}$ in the expiratory phase with normalization during inspiration are considered pathognomonic for celiac artery compression. But this finding only proves that there is compression of the celiac trunk; it does not imply clinical correlation with symptoms.

\section{Excluding other foregut conditions}

MALS is considered a diagnosis of exclusion, and therefore, testing to exclude other foregut pathology should be considered. This may include endoscopy, abdominal imaging (ultrasonography or computed tomography), and functional gastrointestinal studies. Epigastric pain and weight loss can be a manifestation of other disease processes such as biliary colic, gastroparesis, and peptic ulcer disease that should be excluded.

\section{SURGERY DOES NOT ALWAYS CURE}

At my institution, we have strongly advocated for celiac plexus blockade as a diagnostic

\section{Patients with chronic abdominal pain can benefit from a multi- disciplinary evaluation}


modality and predictor of success. It is a very important tool that can help the patient and clinician make a decision toward proceeding with surgical intervention. Nonresponders tend to have a lower likelihood of clinical success after surgery. ${ }^{4}$

It is very important for physicians to have proper conversations with patients and explain the potential risks of the operation, as well as the anticipated outcome. Technical success does not always correlate with clinical success, and $15 \%$ to $25 \%$ of patients can have persistent symptoms despite complete release and neurolysis. ${ }^{4}$

Bleeding during surgery from injury to a major vessel is the most feared complication, and it can be very difficult to manage during minimally invasive approaches. It is the most common reason for conversion to open surgery and can ultimately result in major morbidity and, potentially, death.

\section{KEY CONSIDERATIONS IN THE EVALUATION}

It is important for clinicians evaluating patients with abdominal pain to understand that an imaging finding of celiac artery compression is not diagnostic of MALS. This syndrome requires careful and meticulous evaluation to rule out more common pathology.

Patients suffering from chronic abdominal pain are often exposed to a long course of nondiagnostic testing and missed diagnosis, which typically results in extreme frustration and a sense of hopelessness. As in many cases of chronic illness, these patients benefit from a multidisciplinary evaluation that typically includes a medical specialist, chronic pain specialist, psychologist, and, ultimately, a surgeon with expertise in the field.

\section{DISCLOSURES}

The author reports no relevant financial relationships which, in the context of his contributions, could be perceived as a potential conflict of interest.

\section{REFERENCES}

1. Lipshutz B. A composite study of the coeliac axis artery. Ann Surg 1917; 65(2):159-169. doi:10.1097/00000658-191702000-00006

2. Harjola PT. A rare obstruction of the coeliac artery: Report of a case. Ann Chir Gynaecol Fenn 1963; 52:547-550. pmid:14083857

3. El-Hayek KM, Titus J, Bui A, Mastracci T, Kroh M. Laparoscopic median arcuate ligament release: are we improving symptoms? J Am
Coll Surg 2013; 216(2):272-279. doi:10.1016/j.jamcollsurg.2012.10.004

4. Weber J, Boules $\mathbf{M}$, Fong $\mathrm{K}$, et al. Median arcuate ligament syndrome is not a vascular disease. Ann Vasc Surg 2016; 30:22-27. doi:10.1016/j.avsg.2015.07.013

Address: John H. Rodriguez, MD, FACS, Director of Surgical Endoscopy, Digestive Disease and Surgery Institute, A100, Cleveland Clinic, 9500 Euclid Avenue, Cleveland, $\mathrm{OH} 44195$; rodrigj3@ccf.org 